\title{
Investigation of Dynamics of Macroeconomy and Commodity Mutual Funds: Empirical Evidence from India
}

\author{
Sonali Agarwal', Jwaad Akhtar Khan² \\ ${ }^{1}$ Department of Management, Netaji Subhash University of Technology (Formerly Netaji Subhash Institute of Technology), \\ Dwarka, India \\ ${ }^{2}$ School of Management and Business Studies, Jamia Hamdard University Hamdard Nagar, New Delhi, India \\ Email: sonali1600@yahoo.in, jwaad.a.khan@gmail.com
}

How to cite this paper: Agarwal, S. and Khan, J.A. (2019) Investigation of Dynamics of Macroeconomy and Commodity $\mathrm{Mu}$ tual Funds: Empirical Evidence from India. Theoretical Economics Letters, 9, 872-894. https://doi.org/10.4236/tel.2019.94057

Received: January 27, 2019

Accepted: April 12, 2019

Published: April 15, 2019

Copyright ( 2019 by author(s) and Scientific Research Publishing Inc. This work is licensed under the Creative Commons Attribution International License (CC BY 4.0).

http://creativecommons.org/licenses/by/4.0/

(c) (i) Open Access

\begin{abstract}
Researchers discuss the effect of macroeconomics on the stock market but usually, effects on portfolios like the mutual funds are not very popular. In this research, an attempt was made to study the same. A longitudinal, exploratory study was performed taking three year daily (net asset value) NAVs of 41 mutual funds belonging to two categories (gold funds and energy funds) and series of 9 macroeconomic variables and analysed using time series methods to judge and highlight their impact on the chosen funds. Different macroeconomic variables were found to affect the two categories of funds differently. Investment in energy funds increased whenever there was an increase in money supply. Interest rates had a positive impact on gold mutual fund net asset values. Own fund information was found to have the major impact when excited with one standard deviation shock. The causality between various mutual funds and macroeconomic variables was established. The study highlighted the need to understand the global scenario by both the investor and the mutual fund manager. Since the macroeconomic variables interplay and affect the mutual funds, their understanding would help in stitching more profitable schemes.
\end{abstract}

\section{Keywords}

Macroeconomic Variables, Gold Mutual Funds, Energy Mutual Funds, USD Exchange Rate, Crude Oil Price, Interest Rate

\section{Introduction}

Indian economy is insulated from the foreign world to some extent as the in- 
vestments abroad are less. The investments of FIIs and FDIs are also less as compared to the massive investments by Indian investors. Indian Rupee has been depreciating for quite some time now, but the efforts are on to stabilize this fall. Currency appreciation, depreciation, devaluation etc. all depend on the market forces i.e. supply and demand. If the currency depreciates it benefits the exporters and if it appreciates it benefits the importers. RBI can also control the exchange rates to some extent by the use of foreign currency reserves. Whenever there is some rumor in the market arousing suspicion for the performance of companies, or there is some corporate disclosure, or some movement in the global markets, the investors start buying or selling shares aggressively thus affecting the stock markets. Government policies, national and international events, natural calamities, wars etc. all influence the market movements, investments, exports, and imports. The investor perceptions change very quickly based on the experience of other people. Thus it is important to know what all factors will affect the performance of stocks and in our study the performance of mutual funds.

In the above backdrop, we define the objectives of our research as follows:

- To examine the effect of various macroeconomic factors on mutual funds' performance and their relational outcome.

- To establish the causality between various macroeconomic variables and the select mutual funds.

\section{Literature Review}

From previous researches, it is evident that mutual funds' performance is affected by macroeconomic factors and investor sentiments. The interplay of these governs the actual investment volume. With the change in exchange rate, inflation rate, interest rates etc., the common investor becomes skeptical about the economic condition of the country and withholds his decisions regarding investments.

Ahmad et al. [1] explored the long term and short term causal relationship between NASDAQ, Nikkei, NSE and BSE stock exchanges using Johansen cointegration and Granger causality tests. It was found that there was the absence of long-term co-movement and relationship between the Indian and the US/ Japanese stock markets.

An attempt to understand the concept of mutual funds was made by Gera [2] and he explained its types and benefits. He identified the population, global market movement, GDP composition, inflation and composition of financial savings as macroeconomic factors affecting mutual fund industry in India.

Dash and Kumar [3] studied the effect of select macroeconomic variables upon the Indian mutual fund schemes' returns and volatility. A huge percentage of sample schemes were found to be insensitive to the selected macroeconomic variables, which showed that some other macroeconomic variables are also influencing them which need to be included in future studies. 
Gupta and Siddiqui [4] examined the effect of select macroeconomic factors on Indian stock market with respect to the manner of information absorption.

Srivastava [5] studied the effect of macroeconomic variables on Indian stock market. It was found that IIP, interest rate and WPI had a relatively more significant impact on long-term stock market pricing in India.

Shukla [6] introduced the importance of macroeconomic factors that give rise to risks and uncertainty. He evaluated the interrelationship of macroeconomic variables and AUM of mutual funds.

Khalid [7] studied the long-term co-movement of macroeconomic variables (inflation, exchange rate \& Treasury bill) and Karachi stock exchange return (KSE100). It was found that there was an absence of significant correlation among variables and no evidence of cointegration of variables with share prices existed.

Menike 2006 (as cited in Khalid [7]) performed a study on Sri Lankan stock exchange using multivariate regression model. The monthly data for the period 1991 to 2002 was used. It was found that inflation rate, interest rate, and money supply caused great impact on stock prices.

Patel [8] investigated the effects of selected macroeconomic determinants on Indian stock market performance. A long run relation was found between stock market indices and macroeconomic variables.

Joshi [9] explained the Indian market structure and made an extensive \& systematic study of factors affecting stock market movements. Opinion survey involving personal interviews of 56 stockbrokers, sub-brokers, and their employees was done.

Singhania and Anchalia [10] studied the effect of the financial global crisis on the stock returns volatility of Hong Kong, China, Japan and India using EGARCH. The time series analysis showed that the crisis had a positive impact on returns of Japan, India, and China. Volatility clustering, asymmetry, persistence and leverage effects were also noticed in all the stock return series.

Abdul and Sarvani [11] studied the macroeconomic factors affecting select Indian equity funds and appraise the mutual funds' performance using Sharpe and Treynor models. HDFC top 200 fund \& BNP Paribas equity fund, Franklin India Prima plus fund, UTI mid-cap fund and ICICI Prudential tax plan-(R) emerged the top performers in the various chosen categories of equity schemes.

Based on some previously hypothesized macroeconomic variables, Burmeister et al., 1986-88 (as cited in Abdul and Sarvani [11]) used a multi-index model set and found five variables which according to their research were sufficient to describe any security's return.

Marshall, 1992 (as cited in Abdul and Sarvani [11]) found that real fluctuations in the economy, monetary fluctuations or both had a negative effect on stock returns due to the effect on inflation.

Kumar and Gautam [12] studied the effect of FII \& volatility of other stock exchanges (FTSE 100, NIKKEI 225 \& NASDAQ 100) on BSE volatility. The researchers suggested taking NIFTY stock exchange or blocks of stock exchanges 
for future analysis. The studies can also include long term, short term analysis and various other statistical models like GARCH, ARCH, ARIMA, ARMA etc.

Sanningammanavara et al. [13] studied the influence of eight economic indicators [(GDP) growth rate, exchange rate, gross capital formation (GCF), unemployment rate, inflation rate, gross domestic savings (GDS), interest rate and net (FDI)] on the performance of the stock market and propose a model for it. It was concluded that economic indicators affect stock market to an extent of $77.20 \%$.

Singh [14] assessed the impact and causal relationship between macroeconomic variables and the Indian stock market (BSE Sensex and S \& P CNX Nifty). Industrial production index, money supply (M3), trade deficit, exchange rate. Gold price, crude oil price, wholesale price index. 91 day T-bill (as interest rate proxy IR) and foreign institutional investment (FII) were taken as independent variables. It was concluded that investors were more interested in gold rather than security investment.

Granger causality tests, vector error correction model, and Engle-granger cointegration tests were used by Kwon and Shin, 1999 (as cited in Singh [14]) to test macroeconomic variables' relationship with Korean stock market index.

A long run relationship between selected macroeconomic variables (foreign reserves, inflation, exports, interest rates and industrial production) and Jordanian stock prices was investigated by Maghayereh, 2003 (as cited in Singh, [14]) using monthly time series data.

Erdogan and Ozlale, 2005 (as cited in Singh [14]) performed a study to assess the relationship between Turkey stock returns and macroeconomic variables. It was concluded that exchange rates and Industrial production positively affected stock returns while circulation in money (M1) showed insignificant influence on stock returns.

Short and long-term interest rates, exchange rates, money supply, inflation rate, GDP and domestic retail oil prices were chosen as variables to examine their effect on New Zealand stock prices by Gan et al., 2006 (as cited in Singh, [14]). A significant long-run relationship was revealed. Granger causality tests highlighted that causality did not run from Stock exchange to macroeconomic variables.

The impact of various macroeconomic variables like Industrial production index, money supply, exchange rates, wholesale price index and treasury bill rates on Indian BSE Sensex was studied by Naik and Padhi, 2012 (as cited in Singh [14]). Insignificant association of interest rates \& exchange rates with stock prices was reported.

A study was performed by Sireesha, 2013 (as cited in Singh [14]) to test the effect of macroeconomic variables on Indian stock market index Nifty, silver, and gold prices. Inflation and GDP were found to influence stock returns while gold was affected by money supply.

Mishra and Gupta, 2014 (as cited in Singh [14]) studied the factors responsible for movement in Indian stock market (BSE Sensex). For this IIP, interest 
rate, WPI and MSCI were chosen as independent macroeconomic variables. A positive relation was found between Sensex and the chosen variables.

In their study, Choudhary et al. [15] used non-linear cointegration and causality tests to explore the influence of Indian copper price volatility on Sensex, exchange rate and international copper prices.

A study by Mohanasundari and Vidhyapriya [16] from 2011-2014 explored investor's perception about tax saving mutual fund schemes and its effect on the selection of schemes. The study identified economic factors, regulatory \& management factors and external factors.

Since we are working on mutual funds, we wish to find out that what happens when the global stage is set on fire, or more so what happens when some of the variables of macro economy are changed. How does the market responds to this sudden change? From the previous studies, it has also been seen that various macroeconomic variables are inter-related. The change in one can lead to a drastic change in other. The common investor should be aware of these variables and must know how they interplay and seep into the stock markets and destroy their investments. We here perform this study taking few macroeconomic variables and all the gold and energy mutual funds of India.

\section{Research Design}

The study is exploratory and causal as it first tries to find out the influence of macroeconomic variables on mutual funds and then tries to establish causality between them. The study takes three years of data for study and performs a longitudinal analysis. We took Gold and Energy mutual funds for analysis. The reason is the "hedging effect" i.e. when the stock markets tumble, investors look towards alternate investment avenues. Also, Gold has always been the safe haven for parking money. In the current scenario, energy funds are also doing well and are good investment avenues. The other reason for selecting these funds was that we wanted to study the effect of macroeconomic variables on the mutual funds. Commodities are the most affected avenues as far as macroeconomic shocks are concerned. Hence we selected commodity mutual funds. Since it is difficult to cover all the commodities, we took the most volatile commodity funds which happen to be gold and energy mutual funds.

\subsection{Sample Selection}

We compiled the NAV series of forty-one mutual funds from Mutualfundindia.com website and Bloomberg database. The time period of study was three years from January 2013 to January 2016 highlighting the fact that the study is longitudinal in nature. Fourteen of these were the gold mutual funds, 12 were gold exchange-traded funds and 15 were energy mutual funds (chosen because literature review showed that no work has been done previously on these categories of funds). Various macroeconomic variables like US dollar exchange rate, crude oil price, gold price, silver price, money supply, foreign exchange reserves, interest rate, Sensex and Nifty have been chosen for analysis. For the conveni- 
ence of analysis, all the funds were numbered from $\mathrm{f} \_1$ to $f \_41$ and all the macroeconomic variables taken were numbered from M_1 to M_9 (names and groups in Table A1 and Table A2 in Appendix).

\subsection{Research Hypothesis}

Based on our research objectives, we framed a few hypothesis to verify with our research.

$\mathrm{Ho}_{1}$ : USD exchange rate does not have a significant effect on mutual fund net asset value.

$\mathrm{Ho}_{2}$ : Crude oil price does not have a significant effect on mutual fund net asset value.

$\mathrm{Ho}_{3}$ : Gold price does not have a significant effect on mutual fund net asset value.

$\mathrm{Ho}_{4}$ : Silver price does not have a significant effect on mutual fund net asset value.

$\mathrm{Ho}_{5}$ : Money supply does not have a significant effect on mutual fund net asset value.

$\mathrm{Ho}_{6}$ : foreign exchange reserves do not have a significant effect on mutual fund net asset value.

$\mathrm{Ho}_{7}$ : Interest rate does not have a significant effect on mutual fund net asset value.

$\mathrm{Ho}_{8}$ : Sensex does not have a significant effect on mutual fund net asset value.

$\mathrm{Ho}_{9}$ : Nifty does not have a significant effect on mutual fund net asset value.

For justification of the above stated hypothesis, we followed non-stationary time series analysis pathway. We began with unit root test for checking the non stationarity, followed by cointegration tests and vector error correction models to establish the cointegration direction and strength. The series that possessed cointegration were further tested for short term causality using granger causality test. Then the results from all the above analysis were further confirmed by giving impulses of own series and all the macroeconomic variables. These impulses were of one standard deviation equivalent. The results of impulse response were desicated by variance decomposition method. Here it was seen that how much variation in NAV series was caused due to which impulse.

\section{Methodology}

The methodology starts with the application of unit root test for stationarity. After performing unit root test, we see that if the series are non-stationary, then they should get stationary at the same level after differencing. If they get stationary at the same level, then we can say that they are integrated of that order and can proceed further with cointegration. For getting the cointegration equations, we apply vector error correction mechanism (VECM) and construct error correction equations. The coefficients of these equations tell about the speed of adjustment of the variable. To see short-run relationship, we perform Granger causality which is a concept based on the idea of succession in time. To make the 
results more evidential, we next give impulse of macroeconomic variables and funds information to the fund value. To see how much shock is coming from the exogenous variables and how much from the fund information itself, we decompose the variation caused and see which variable has caused how much variance in the fund value. We have followed these steps and have framed the results with respect to all the tests conducted.

\section{Proposed Model}

The mutual fund net asset prices and the various macroeconomic variables are implicitly depicted as:

$$
\text { NAV = f(USD, OIL, GLD, SLVR, MS, FER, IR, SENSEX, NIFTY })
$$

where, NAV refers to the net asset value of the chosen mutual fund and the right hand side variables are the US dollar exchange rate with Indian rupee (USD), crude oil price (OIL), gold price (GLD), silver price (SLVR), money supply in the country (MS), foreign exchange reserves (FER), interest rate (IR), Sensex and Nifty indices.

\section{Data Analysis Results}

\subsection{Stationarity Test}

We performed stationarity test (Table 1 and Table 2 ) to see whether our series are stationary or non-stationary. When Augmented Dickey Fuller Unit root stationarity test was performed at level, the absolute value of ADF test statistics for all the mutual funds and the macroeconomic variables were found to be less than the absolute critical values at one percent and five percent levels of significance. The p-values were found to be higher than the levels of significances, hence we accept Ho, i.e. the series have unit roots and are thus non-stationary. The number of lags for the test have been selected on the basis of Schwarz criterion. From the tables, it can also be seen that the ADF statistics were more than the absolute critical values at one percent and five percent levels of significance when the ADF test was performed after taking first difference for all the sample series. Also, the p-value (0.000) was less than 0.05 , which shows rejection of the null hypothesis. Thus the series are now stationary at first difference and are said to be integrated of order I.

\subsection{Cointegration Test}

Since all the series were found to be integrated of order 1, we performed Johansen cointegration test for each group. Every group consisted of one mutual fund and all the nine macroeconomic variables. The trace test and the maximum eigen-value test indicated a different number of cointegrating equations in each group.

It was seen that according to Johansen cointegration test, each group contains cointegrating equations. The output of the test was used to find vector error correction equation to know the error correction speed whenever the cointegrating series are disturbed due to shock. 
Table 1. ADF test of mutual fund series.

\begin{tabular}{|c|c|c|c|c|c|c|c|c|c|c|}
\hline $\begin{array}{l}\text { Mutual } \\
\text { fund }\end{array}$ & $\begin{array}{c}\text { ADF } \\
\text { statistic } \\
\text { at level }\end{array}$ & $\begin{array}{l}\text { Test critical } \\
\text { values at } \\
1 \% \text { level of } \\
\text { significance }\end{array}$ & $\begin{array}{c}\text { Test critical } \\
\text { values at } \\
5 \% \text { level of } \\
\text { significance }\end{array}$ & $\begin{array}{c}\text { Test critical } \\
\text { values at } \\
10 \% \text { level of } \\
\text { significance }\end{array}$ & $\mathrm{P}$-value & $\begin{array}{c}\text { ADF } \\
\text { statistic at } \\
1^{\text {st }} \text { difference }\end{array}$ & $\begin{array}{c}\text { Test critical } \\
\text { values at } \\
1 \% \text { level of } \\
\text { significance }\end{array}$ & $\begin{array}{c}\text { Test critical } \\
\text { values at } \\
5 \% \text { level of } \\
\text { significance }\end{array}$ & $\begin{array}{c}\text { Test critical } \\
\text { values at } \\
10 \% \text { level of } \\
\text { significance }\end{array}$ & $P$-value \\
\hline f_1 & -2.771123 & -3.544063 & -2.910860 & -2.593090 & 0.068500 & -8.343738 & -3.546099 & -2.911730 & -2.593551 & 0.000000 \\
\hline f_2 & -1.518975 & -3.544063 & -2.910860 & -2.593090 & 0.517300 & -7.743742 & -3.546099 & -2.911730 & -2.593551 & 0.000000 \\
\hline f_3 & -2.184721 & -3.544063 & -2.910860 & -2.593090 & 0.213900 & -8.101964 & -3.546099 & -2.911730 & -2.593551 & 0.000000 \\
\hline f_4 & -2.558827 & -3.544063 & -2.910860 & -2.593090 & 0.107200 & -9.394846 & -3.546099 & -2.911730 & -2.593551 & 0.000000 \\
\hline f_5 & -2.009690 & -3.544063 & -2.910860 & -2.593090 & 0.282000 & -7.601256 & -3.546099 & -2.911730 & -2.593551 & 0.000000 \\
\hline f_6 & -2.265115 & -3.544063 & -2.910860 & -2.593090 & 0.186500 & -8.062592 & -3.546099 & -2.911730 & -2.593551 & 0.000000 \\
\hline f_7 & -2.869932 & -3.544063 & -2.910860 & -2.593090 & 0.054900 & -5.839286 & -3.568308 & -2.921175 & -2.598551 & 0.000000 \\
\hline f_8 & -1.322064 & -3.544063 & -2.910860 & -2.593090 & 0.613900 & -7.804268 & -3.546099 & -2.911730 & -2.593551 & 0.000000 \\
\hline f_9 & -2.050167 & -3.544063 & -2.910860 & -2.593090 & 0.265300 & -8.206944 & -3.546099 & -2.911730 & -2.593551 & 0.000000 \\
\hline f_10 & -2.153083 & -3.544063 & -2.910860 & -2.593090 & 0.225400 & -8.666955 & -3.546099 & -2.911730 & -2.593551 & 0.000000 \\
\hline f_11 & -2.064209 & -3.544063 & -2.910860 & -2.593090 & 0.259600 & -8.493626 & -3.546099 & -2.911730 & -2.593551 & 0.000000 \\
\hline f_12 & -2.165960 & -3.544063 & -2.910860 & -2.593090 & 0.227100 & -7.332969 & -3.546099 & -2.911730 & -2.593551 & 0.000000 \\
\hline f_13 & -2.690280 & -3.544063 & -2.910860 & -2.593090 & 0.081600 & -7.139850 & -3.546099 & -2.911730 & -2.593551 & 0.000000 \\
\hline f_14 & -2.127609 & -3.544063 & -2.910860 & -2.593090 & 0.234900 & -8.309649 & -3.546099 & -2.911730 & -2.593551 & 0.000000 \\
\hline f_15 & -2.049778 & -3.544063 & -2.910860 & -2.593090 & 0.066000 & -5.787755 & -3.568308 & -2.921175 & -2.598551 & 0.000000 \\
\hline f_16 & -2.033060 & -3.544063 & -2.910860 & -2.593090 & 0.067500 & -5.770883 & -3.568308 & -2.921175 & -2.598551 & 0.000000 \\
\hline f_17 & -2.765677 & -3.544063 & -2.910860 & -2.593090 & 0.069300 & -8.437351 & -3.546099 & -2.911730 & -2.593551 & 0.000000 \\
\hline f_18 & -2.648665 & -3.544063 & -2.910860 & -2.593090 & 0.089100 & -8.130755 & -3.546099 & -2.911730 & -2.593551 & 0.000000 \\
\hline f_19 & -2.074736 & -3.544063 & -2.910860 & -2.593090 & 0.073900 & -5.791633 & -3.568308 & -2.921175 & -2.598551 & 0.000000 \\
\hline f_20 & -2.034451 & -3.544063 & -2.910860 & -2.593090 & 0.057400 & -5.782584 & -3.568308 & -2.921175 & -2.598551 & 0.000000 \\
\hline f_21 & -2.035310 & -3.544063 & -2.910860 & -2.593090 & 0.067300 & -8.050313 & -3.546099 & -2.911730 & -2.593551 & 0.000000 \\
\hline f_22 & -2.059742 & -3.544063 & -2.910860 & -2.593090 & 0.065100 & -5.794623 & -3.568308 & -2.921175 & -2.598551 & 0.000000 \\
\hline f_23 & -2.889992 & -3.544063 & -2.910860 & -2.593090 & 0.052500 & -8.445508 & -3.546099 & -2.911730 & -2.593551 & 0.000000 \\
\hline f_24 & -2.017811 & -3.544063 & -2.910860 & -2.593090 & 0.058900 & -5.785990 & -3.568308 & -2.921175 & -2.598551 & 0.000000 \\
\hline f_25 & -2.078674 & -3.544063 & -2.910860 & -2.593090 & 0.063600 & -8.033548 & -3.546099 & -2.911730 & -2.593551 & 0.000000 \\
\hline f_26 & -2.023827 & -3.544063 & -2.910860 & -2.593090 & 0.058300 & -8.020354 & -3.546099 & -2.911730 & -2.593551 & 0.000000 \\
\hline f_27 & -1.004166 & -3.544063 & -2.910860 & -2.593090 & 0.746500 & -7.659445 & -3.546099 & -2.911730 & -2.593551 & 0.000000 \\
\hline f_28 & -0.704624 & -3.544063 & -2.910860 & -2.593090 & 0.837400 & -7.334186 & -3.546099 & -2.911730 & -2.593551 & 0.000000 \\
\hline f_29 & -0.670113 & -3.544063 & -2.910860 & -2.593090 & 0.846000 & -7.791967 & -3.546099 & -2.911730 & -2.593551 & 0.000000 \\
\hline f_30 & -0.646962 & -3.544063 & -2.910860 & -2.593090 & 0.851600 & -7.039387 & -3.546099 & -2.911730 & -2.593551 & 0.000000 \\
\hline f_31 & -0.255373 & -3.544063 & -2.910860 & -2.593090 & 0.924700 & -6.754967 & -3.546099 & -2.911730 & -2.593551 & 0.000000 \\
\hline f_32 & -0.945031 & -3.544063 & -2.910860 & -2.593090 & 0.767000 & -7.357812 & -3.546099 & -2.911730 & -2.593551 & 0.000000 \\
\hline f_33 & -0.663167 & -3.544063 & -2.910860 & -2.593090 & 0.847700 & -7.690004 & -3.546099 & -2.911730 & -2.593551 & 0.000000 \\
\hline f_34 & -0.621744 & -3.544063 & -2.910860 & -2.593090 & 0.857500 & -6.824495 & -3.546099 & -2.911730 & -2.593551 & 0.000000 \\
\hline f_35 & -0.368118 & -3.544063 & -2.910860 & -2.593090 & 0.907400 & -6.875377 & -3.546099 & -2.911730 & -2.593551 & 0.000000 \\
\hline f_36 & -0.677402 & -3.544063 & -2.910860 & -2.593090 & 0.844200 & -6.868468 & -3.546099 & -2.911730 & -2.593551 & 0.000000 \\
\hline f_37 & -0.817272 & -3.544063 & -2.910860 & -2.593090 & 0.806800 & -7.079072 & -3.546099 & -2.911730 & -2.593551 & 0.000000 \\
\hline f_38 & -0.586640 & -3.544063 & -2.910860 & -2.593090 & 0.865400 & -6.536958 & -3.546099 & -2.911730 & -2.593551 & 0.000000 \\
\hline f_39 & -0.690882 & -3.544063 & -2.910860 & -2.593090 & 0.840900 & -7.935993 & -3.546099 & -2.911730 & -2.593551 & 0.000000 \\
\hline f_40 & -0.793372 & -3.544063 & -2.910860 & -2.593090 & 0.813600 & -7.396123 & -3.546099 & -2.911730 & -2.593551 & 0.000000 \\
\hline f_41 & -0.507871 & -3.544063 & -2.910860 & -2.593090 & 0.881900 & -6.252981 & -3.546099 & -2.911730 & -2.593551 & 0.000000 \\
\hline
\end{tabular}


Table 2. ADF test of macroeconomic series.

\begin{tabular}{|c|c|c|c|c|c|c|c|c|c|c|}
\hline $\begin{array}{c}\text { Macroeconomic } \\
\text { variable }\end{array}$ & $\begin{array}{c}\text { ADF } \\
\text { statistic } \\
\text { at level }\end{array}$ & $\begin{array}{c}\text { Test } \\
\text { critical } \\
\text { values at } 1 \% \\
\text { level of } \\
\text { significance }\end{array}$ & $\begin{array}{l}\text { Test critical } \\
\text { values at } \\
5 \% \text { level of } \\
\text { significance }\end{array}$ & $\begin{array}{c}\text { Test critical } \\
\text { values at } \\
10 \% \text { level of } \\
\text { significance }\end{array}$ & P-value & $\begin{array}{l}\text { ADF statistic } \\
\text { at } 1^{\text {st }} \\
\text { difference }\end{array}$ & $\begin{array}{c}\text { Test critical } \\
\text { values at } \\
1 \% \text { level of } \\
\text { significance }\end{array}$ & $\begin{array}{l}\text { Test critical } \\
\text { values at } \\
5 \% \text { level of } \\
\text { significance }\end{array}$ & $\begin{array}{l}\text { Test critical } \\
\text { values at } \\
10 \% \text { level of } \\
\text { significance }\end{array}$ & P-value \\
\hline M_1 & -2.771123 & -3.544063 & -2.910860 & -2.593090 & 0.068500 & -8.343738 & -3.546099 & -2.911730 & -2.593551 & 0.000000 \\
\hline M_2 & -1.518975 & -3.544063 & -2.910860 & -2.593090 & 0.517300 & -7.743742 & -3.546099 & -2.911730 & -2.593551 & 0.000000 \\
\hline M_3 & -2.184721 & -3.544063 & -2.910860 & -2.593090 & 0.213900 & -8.101964 & -3.546099 & -2.911730 & -2.593551 & 0.000000 \\
\hline M_4 & -2.558827 & -3.544063 & -2.910860 & -2.593090 & 0.107200 & -9.394846 & -3.546099 & -2.911730 & -2.593551 & 0.000000 \\
\hline M_5 & -2.009690 & -3.544063 & -2.910860 & -2.593090 & 0.282000 & -7.601256 & -3.546099 & -2.911730 & -2.593551 & 0.000000 \\
\hline M_6 & -2.265115 & -3.544063 & -2.910860 & -2.593090 & 0.186500 & -8.062592 & -3.546099 & -2.911730 & -2.593551 & 0.000000 \\
\hline M_7 & -2.869932 & -3.544063 & -2.910860 & -2.593090 & 0.054900 & -5.839286 & -3.568308 & -2.921175 & -2.598551 & 0.000000 \\
\hline M_8 & -1.322064 & -3.544063 & -2.910860 & -2.593090 & 0.613900 & -7.804268 & -3.546099 & -2.911730 & -2.593551 & 0.000000 \\
\hline M_9 & -2.050167 & -3.544063 & -2.910860 & -2.593090 & 0.265300 & -8.206944 & -3.546099 & -2.911730 & -2.593551 & 0.000000 \\
\hline
\end{tabular}

\subsection{VECM and Equation Estimation}

Equations were found after seeing the significance of the constant terms and the probability value. The value of the constants was then noted and the equations formed. The following equations were formed:

$$
\begin{aligned}
& D\left(f \_1\right)=-0.70 * D\left(f \_1(-2)\right)+130.54 * D\left(M_{-} 7(-1)\right) \\
& D\left(f \_2\right)=0.36 * D\left(M \_7(-1)\right) \\
& D\left(f \_3\right)=0.38 * D\left(M \_7(-1)\right) \\
& D\left(f \_4\right)=-0.51 * D\left(f \_4(-1)\right)+0.39 * D\left(M \_7(-1)\right) \\
& D\left(f \_5\right)=-0.56 * D\left(f \_5(-2)\right)+0.35 * D\left(M_{-} 7(-1)\right) \\
& D\left(f \_6\right)=-0.49 * D\left(f \_6(-2)\right)+0.34 * D\left(M_{-} 7(-1)\right) \\
& D\left(f \_7\right)=0.38 E-05 * D\left(M \_6(-1)\right)+0.46 * D\left(M \_7(-1)\right) \\
& D\left(f \_8\right)=0.30 * D\left(M \_7(-1)\right) \\
& D\left(f \_9\right)=-0.49 * D\left(f \_9(-2)\right)+0.45 * D\left(M \_7(-1)\right) \\
& D\left(f \_10\right)=0.46 * D\left(M \_7(-1)\right) \\
& D\left(f \_11\right)=-0.60 * D\left(f \_11(-2)\right)+0.37 * D\left(M \_7(-1)\right) \\
& D\left(f \_12\right)=0.45 * D\left(M \_4(-1)\right)+0.40 * D\left(M \_4(-2)\right) \\
& +0.01 * D\left(M \_6(-1)\right)+1.05 * D\left(M_{-} 7(-1)\right) \\
& D\left(f \_13\right)=0.37 * D\left(M \_4(-2)\right)+8.31 E-05 * D\left(M \_6(-1)\right) \\
& +0.80 * D\left(M \_7(-1)\right) \\
& D\left(f_{-} 14\right)=0.41 * D\left(M_{-} 7(-1)\right)
\end{aligned}
$$




$$
\begin{aligned}
& D\left(f \_15\right)=0.01 * D\left(M \_6(-1)\right)+68.35 * D\left(M \_7(-1)\right) \\
& D\left(f \_16\right)=0.01 * D\left(M \_6(-1)\right)+138.68 * D\left(M \_7(-1)\right) \\
& D\left(f \_17\right)=0.01 * D\left(M \_6(-1)\right)+146.35 * D\left(M \_7(-1)\right) \\
& D\left(f \_18\right)=121.29 * D\left(M \_7(-1)\right) \\
& D\left(f \_19\right)=0.01 * D\left(M \_6(-1)\right)+135.63 * D\left(M \_7(-1)\right) \\
& D\left(f \_20\right)=0.01 * D\left(M \_6(-1)\right)+139.47 * D\left(M \_7(-1)\right) \\
& D\left(f \_21\right)=0.01 * D\left(M \_6(-1)\right)+14.08 * D\left(M \_7(-1)\right) \\
& D\left(f \_22\right)=0.01 * D\left(M \_6(-1)\right)+142.46 * D\left(M \_7(-1)\right) \\
& D\left(f \_23\right)=0.01 * D\left(M \_6(-1)\right)+156.32 * D\left(M \_7(-1)\right) \\
& D\left(f \_24\right)=0.01 * D\left(M \_6(-1)\right)+13.67 * D\left(M \_7(-1)\right) \\
& D\left(f \_25\right)=0.01 * D\left(M \_6(-1)\right)+140.44 * D\left(M_{-} 7(-1)\right) \\
& D\left(f \_26\right)=0.01 * D\left(M \_6(-1)\right)+136.11 * D\left(M \_7(-1)\right) \\
& D\left(f \_29\right)=0.52 * D\left(M \_5(-1)\right) \\
& D\left(f \_30\right)=0.03 *\left(f \_30(-1)-1.70 * M \_1(-1)-3.57 * M \_2(-1)\right. \\
& +0.64 * M \_3(-1)-19.99 * M \_4(-1)+3.45 * M \_5(-1) \\
& +0.01 * M \_6(-1)-17.60 * M \_7(-1)+0.52 * M \_8(-1) \\
& \left.-1.75 * M \_9(-1)-94.57\right)+1.39 \\
& D\left(f \_31\right)=0.04 *\left(f \_31(-1)-1.50 * M \_1(-1)-0.68 * M \_2(-1)\right. \\
& +0.05 * M \_3(-1)-2.23 * M \_4(-1)+0.76 * M \_5(-1) \\
& +7.45 E-05 * M_{-} 6(-1)-1.91 * M_{-} 7(-1) \\
& \left.+0.09 * M \_8(-1)-0.30 * M \_9(-1)+104.75\right) \\
& +0.49 * D\left(f \_31(-2)\right)+0.08 * D\left(M \_2(-2)\right)+0.25 \\
& D\left(f \_32\right)=0.50 * D\left(f \_32(-2)\right) \\
& D\left(f \_33\right)=0.19\left(f \_33(-1)-0.43 * M \_1(-1)-0.08 * M \_2(-1)\right. \\
& +0.01 * M \_3(-1)-0.38 * M \_4(-1)+0.36 * M \_5(-1) \\
& -1.23 E-05 * M \_6(-1)+0.54 * M \_7(-1) \\
& \left.+0.01 * M \_8(-1)-0.04 * M \_9(-1)+39.09\right)+0.17 \\
& D\left(f \_34\right)=0.08 *\left(f \_34(-1)-0.72 * M \_1(-1)-0.39 * M \_2(-1)\right. \\
& +0.04 * M \_3(-1)-1.61 * M \_4(-1)+0.91 * M \_5(-1) \\
& -4.13 E-05 * M \_6(-1)+0.27 * M_{-} 7(-1) \\
& \left.+0.05 * M \_8(-1)-0.18 * M \_9(-1)+64.05\right) \\
& -0.31 * D\left(M \_1(-1)\right)+0.28
\end{aligned}
$$




$$
\begin{aligned}
& D\left(f \_35\right)=0.20 *\left(f \_35(-1)-0.89 * M \_1(-1)-0.21 * M \_2(-1)\right. \\
& +0.02 * M \_3(-1)-1.16 * M \_4(-1)+1.53 * M \_5(-1) \\
& -8.79 E-05 * M_{-} 6(-1)+0.58 * M_{-} 7(-1) \\
& \left.+0.04 * M_{-} 8(-1)-0.14 * M_{-} 9(-1)+101.18\right)+0.55 \\
& D\left(f \_36\right)=0.20 *\left(f \_36(-1)-0.19 * M \_1(-1)-0.16 * M \_2(-1)\right. \\
& +0.03 * M \_3(-1)-1.15 * M \_4(-1)+0.51 * M \_5(-1) \\
& +2.01 E-07 * M_{-} 6(-1)-0.07 * M_{-} 7(-1) \\
& \left.+0.02 * M \_8(-1)-0.08 * M \_9(-1)+15.19\right)+0.24 \\
& D\left(f \_37\right)=-0.08 *\left(f \_37(-1)-0.82 * M \_1(-1)+0.21 * M \_2(-1)\right. \\
& -0.08 * M \_3(-1)+1.97 * M_{-} 4(-1)+0.61 * M \_5(-1) \\
& +4.49 * M \_7(-1)-0.04 * M \_8(-1) \\
& \left.+0.14 * M_{-} 9(-1)+122.94\right)+0.94 * D\left(f \_37(-2)\right) \\
& -0.21 * D\left(M \_1(-1)\right)-0.15 \\
& D\left(f \_38\right)=0.04 * D\left(M \_2(-2)\right)+0.16 * D\left(M \_5(-1)\right)+0.12 \\
& D\left(f \_39\right)=0.13 *\left(f \_39(-1)-0.21 * M \_1(-1)-0.10 * M \_2(-1)\right. \\
& +0.01 * M \_3(-1)-0.66 * M \_4(-1)+0.37 * M \_5(-1) \\
& +3.67 E-06 * M_{-} 6(-1)+0.02 * M_{-} 7(-1) \\
& \left.+0.02 * M \_8(-1)-0.06 * M \_9(-1)+10.73\right) \\
& +0.06 * D\left(M \_2(-2)\right)-0.25 * D\left(M \_7(-1)\right)+0.13 \\
& D\left(f \_40\right)=0.14 *\left(f \_40(-1)-1.01 * M \_1(-1)-0.47 * M \_2(-1)\right. \\
& +0.05 * M \_3(-1)-2.10 * M_{-} 4(-1)+1.16 * M \_5(-1) \\
& -3.92 E-05 * M_{-} 6(-1)+0.26 * M_{-} 7(-1) \\
& \left.+0.06 * M \_8(-1)-0.22 * M \_9(-1)+94.42\right)+0.56
\end{aligned}
$$

For the convenience of interpretation, the decimal points have been restricted to 2 in all cases.

From the equations (II-XXXIX) above it can be seen that gold funds were cointegrated with lags of own information and the lags of interest rate \& foreign exchange reserves. In a few cases, cointegration with silver price lags was also seen. The error correction speed was very high when there was a change in interest rate but quite low when there was a change in foreign exchange reserves. Whenever there was a change in own information, the error correction speed was appreciable. The energy funds were found to be dependent on all the macroeconomic variables but the error correction speed was quite low showing a low degree of cointegration. Money supply showed good cointegration with an appreciable error correction speed when energy funds were taken. Crude oil price was also seen cointegrated with energy funds but the error correction speed was low. Own lagged information was found to have a vast impact even up to two lags. 
An important point to note here is that there was no cointegration found with $\mathrm{f} \_27, \mathrm{f} \_28$ and $\mathrm{f} \_41$, all of which happen to be energy funds. Thus we can say that the indication of cointegration as seen with Johansen cointegration test was may be between the various macroeconomic variables themselves and not with these three funds. Also, funds from $f \_15$ to $f \_26$ are exchange-traded funds and it can be seen that they are highly cointegrated with foreign exchange reserves and interest rate, both in their first lags. A change in either of them leads to instant error correction and the speed is high in case of changes in interest rates.

\subsection{Granger Causality}

In Figure 1, Causality was seen running from USD exchange rate to some gold mutual funds. Since less number of samples were found to be granger caused, hence the result cannot be generalized to all the gold funds.

Granger causality ran from foreign exchange reserves to gold mutual funds (Figure 2). Every time when there was a change in foreign exchange reserves of the country, there occurred a change in gold mutual fund NAVs. Thus a good impact.

In Figure 3, Causality also ran from interest rate to all the gold mutual funds. It means that every time there was a change in interest rates, there occurred a change in the net asset value of the funds.

Silver prices were found to Granger cause some of the gold mutual funds (Figure 4). Since less number of samples were found to be granger caused, hence the result cannot be generalized to all the gold funds.

Every time there was a change in nifty index, it was followed by a change in the net asset values of some of the gold mutual funds. But since very less were found to be following, the result cannot be generalized for the entire category of gold funds (Figure 5).

Gold mutual funds and macroeconomic variables.

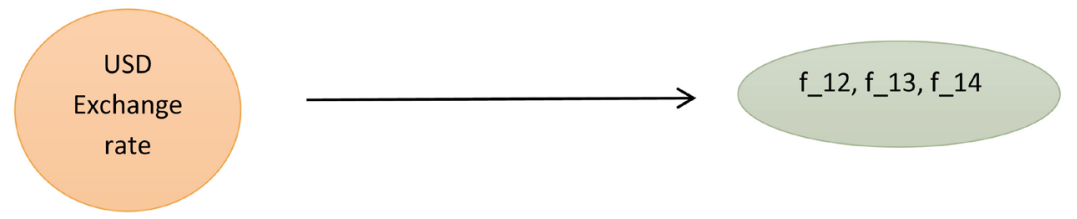

Figure 1. Granger causality 1. (Source: researcher's analysis).

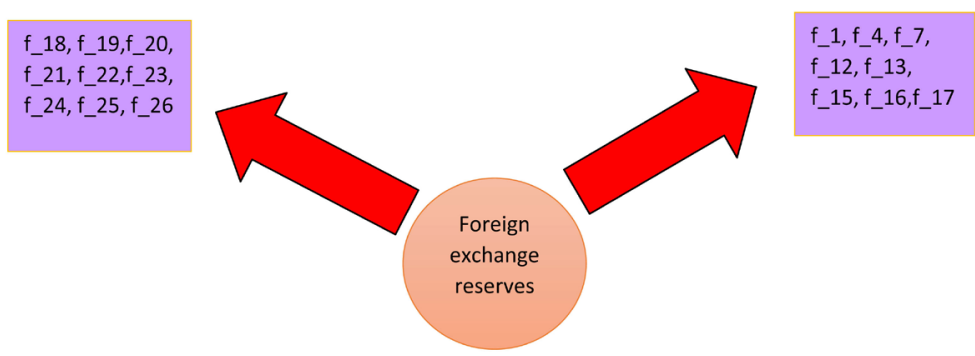

Figure 2. Granger causality 2. (Source: researcher's analysis). 


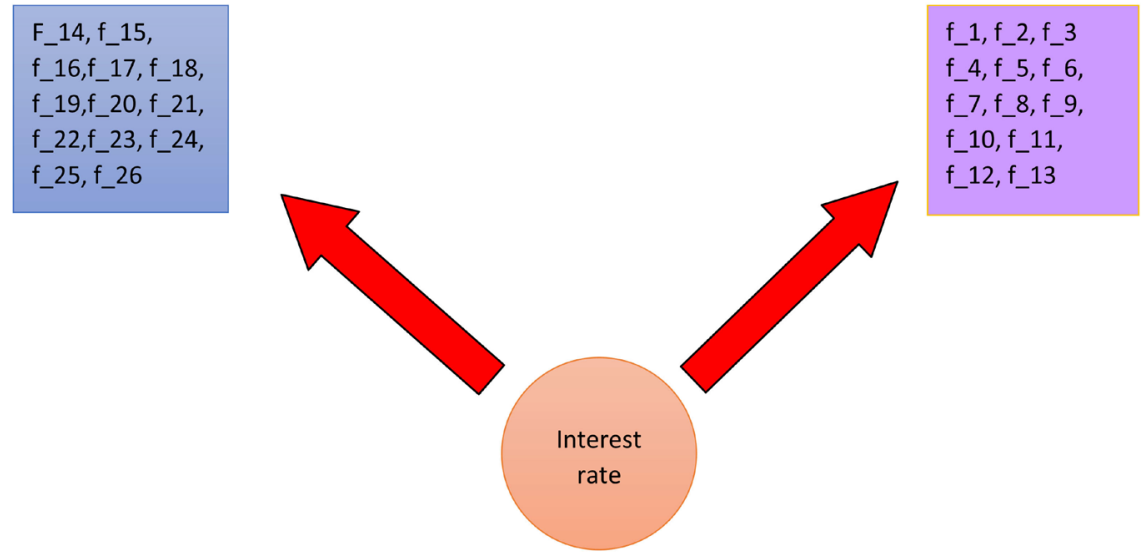

Figure 3. Granger causality 3. (Source: researcher's analysis).
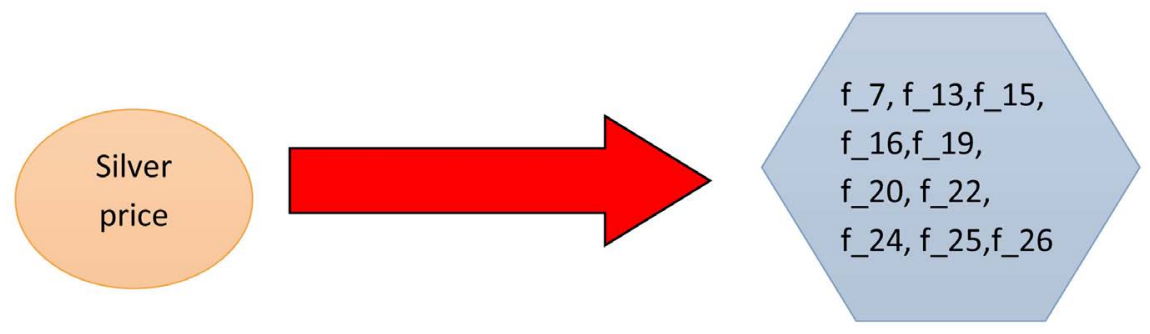

Figure 4. Granger causality 4. (Source: researcher’s analysis).

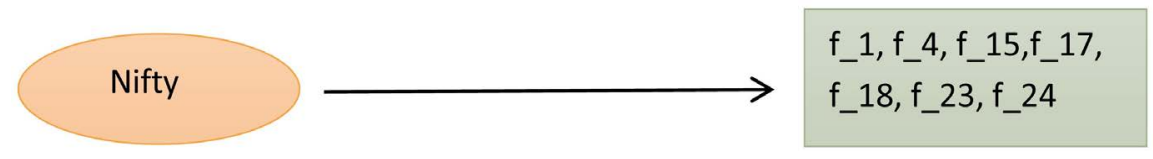

Figure 5. Granger causality 5. (Source: researcher's analysis).

Apart from these, money supply was found to Granger cause f_3 \&f_18, Sensex index was found to Granger cause $\mathrm{f} \_4, \mathrm{f} \_17 \& \mathrm{f} \_18$. Crude oil price granger caused f_13. These latter results, however, could not be taken as an evidence to generalize the causality in the funds.

From Figure 6, it can be seen that Energy funds were found to be granger caused by Sensex. Every time there is a change in Sensex, there occurs a change in net asset value of energy funds.

Nifty (in Figure 7) was also found to Granger cause energy funds. Every time when there is a change in Nifty index, there occurs a change in energy funds.

Figure 8 shows that Change in foreign reserves and money supply of the country led to a change in energy fund net asset values. However, due to less sample response to these variables, the results cannot be generalized for the entire category of energy funds.

Apart from this causality was also seen running from interest rate to $f \_28$, f_36 \& f_39.

Figure 9 showed that Interest rate came out as the major influencer on other macroeconomic variables. It granger caused USD exchange rate, gold price, 
Energy funds and macroeconomic variables.

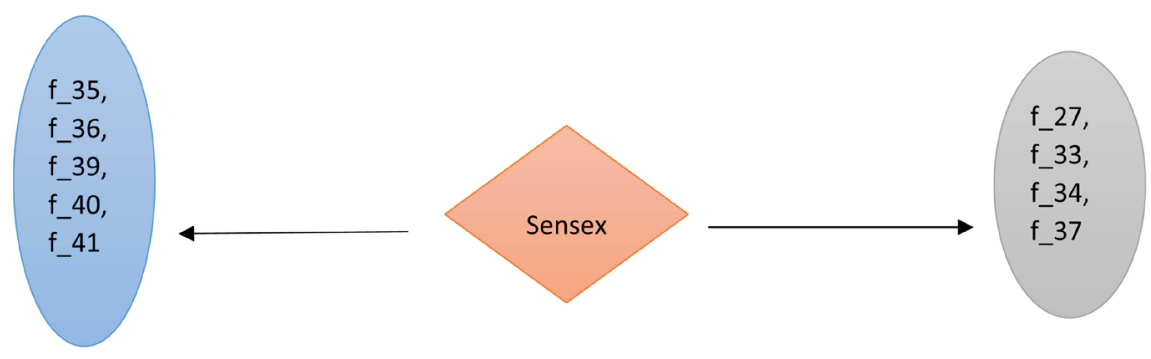

Figure 6. Granger causality 6. (Source: researcher's analysis).

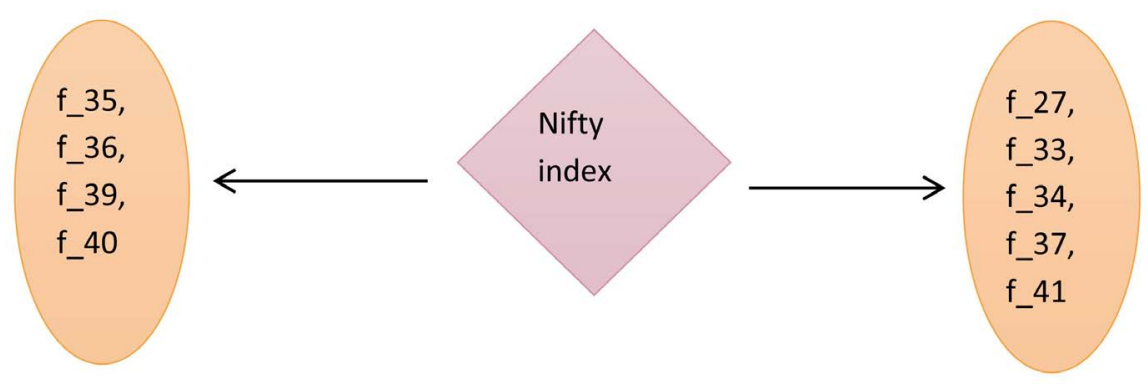

Figure 7. Granger causality 7. (Source: researcher's analysis).

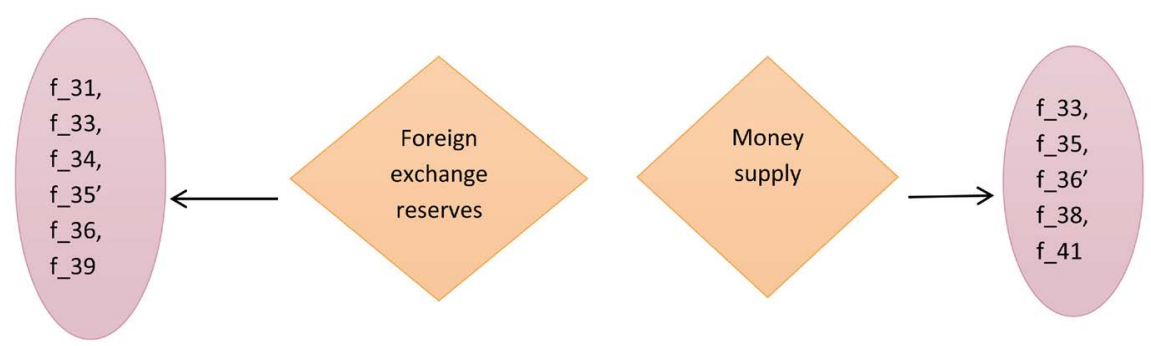

Figure 8. Granger causality 8. (Source: researcher's analysis).

Interplay of macroeconomic variables
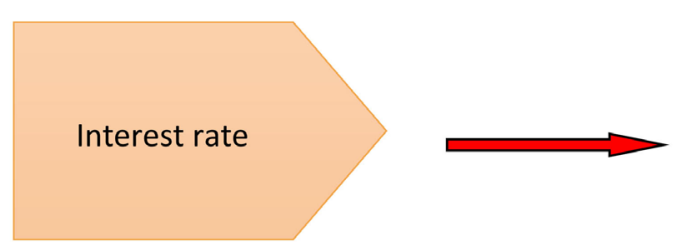

USD exchange rate, gold price, silver price, foreign exchange reserves, Sensex, Nifty

Figure 9. Granger causality 9. (Source: researcher’s analysis).

silver price, foreign exchange reserves, Sensex \& Nifty index. It can thus be taken as the major variable in the macroeconomic stage of our country. Apart from this Sensex and Nifty were found to Granger cause each other. Money supply and Sensex also granger caused each other. Money supply also granger caused Nifty. Gold and silver prices granger caused USD exchange rate. Foreign exchange reserves granger caused gold prices.

From the above Granger causality analysis, it can be said that foreign ex- 
change reserves, interest rate, silver price all influence the gold mutual funds. Nifty index also influenced gold funds in some cases but the result cannot be generalized. Sensex and Nifty influenced energy funds and caused changes. Country's money supply and foreign exchange reserves also influenced energy funds. The interest rate was the major catalyst for changing all the other macroeconomic variables. Sensex \& money supply were inter-related.

\subsection{Impulse Response}

When an impulse of one standard deviation of fund's own NAV's was given, it produced an immediate positive impact to the NAV's in all the funds. In some cases (like in funds $\mathrm{f} \_12, \mathrm{f} \_13, \mathrm{f} \_27, \mathrm{f} \_28, \mathrm{f} \_30, \mathrm{f} \_31, \mathrm{f} \_32, \mathrm{f} \_34, \mathrm{f} \_36, \mathrm{f} \_37, \mathrm{f} \_38$, f_40 \& f_41) the impulse retained its immediate excited value till the end of 10 periods. In rest others, the immediately excited values lowered down to a new value which was much higher than the initial unexcited value.

USD exchange rate shock caused slight negative impact on NAV of $f \_2, f \_3$, $\mathrm{f} \_5, \mathrm{f} \_7, \mathrm{f} \_8, \mathrm{f} \_9, \mathrm{f} \_11, \mathrm{f} \_12, \mathrm{f} \_13, \mathrm{f} \_21 \mathrm{\&} \mathrm{f} \_24$ after an immediate null effect, which subsided to normal after 4 periods. Slight negative non diminishing effects were produced on funds $\mathrm{f} \_27, \mathrm{f} \_28, \mathrm{f} \_29, \mathrm{f} \_30, \mathrm{f} \_31, \mathrm{f} \_32, \mathrm{f} \_33, \mathrm{f} \_34, \mathrm{f} \_36$, $\mathrm{f} \_37, \mathrm{f} \_38$ \& $\mathrm{f} \_41$. A marked negative impact was seen in funds $\mathrm{f} \_1, \mathrm{f} \_15, \mathrm{f} \_16$, $\mathrm{f} \_17, \mathrm{f} \_18, \mathrm{f} \_19, \mathrm{f} \_20, \mathrm{f} \_22, \mathrm{f} \_23, \mathrm{f} \_25 \mathrm{\&} \mathrm{f} \_26$. But in all these latter cases, the effect subsided after four periods. A point worth noting is that, there was no immediate effect of the impulse of USD exchange rate in all the funds.

The crude oil price impact had negligible effect in most of the cases. But funds like f_27, f_29, f_30, f_31, f_32, f_33, f_34, f_36 f_37, f_38, f_39 \& f_41 had a slight negative impact instantly till the $2^{\text {nd }}$ period which subsequently changed into a slight positive impact towards the end of 10 periods.

The gold price shock had a negligible effect on few funds. A small negative impact on NAVs was reported in fund $\mathrm{f} \_12 \& \mathrm{f} \_13$. A small positive impact was seen on f_27, f_30, f_31, f_32, f_34, f_35, f_36, f_37, f_38 \& f_39 which failed to subside over 10 periods. A Marked positive effect was found on NAVs of $f_{-} 1$, f_17 \&f_23 but the effect subsided eventually.

Energy funds recorded no change in NAVs when an innovation of silver price was given. A slight positive diminishing effect was however witnessed in case of few gold funds like f_16, f_19, f_20, f_22, f_23, f_25 \& f_26.

Next, we checked for the introduction of one standard deviation innovation in the money supply of the country. It was found that a marked subsequent negative impact occurred on funds $\mathrm{f} \_1, \mathrm{f} \_15, \mathrm{f} \_16, \mathrm{f} \_17, \mathrm{f} \_18, \mathrm{f} \_19, \mathrm{f} \_20, \mathrm{f} \_22, \mathrm{f} \_23$, $\mathrm{f} \_25 \& \mathrm{f} \_26$ which fluctuated till the $6^{\text {th }}$ period and then subsided. A little negative impact was witnessed in case of f_7, f_21 \& f_24. Energy funds, however, presented a different picture. An increasing positive value of NAVs was seen in funds f_27, f_29, f_30, f_31, f_32, f_33, f_34, f_35, f_36, f_37, f_38, f_39, f_40 \& f_41.

All the funds were exposed to one standard deviation shock in foreign exchange reserves. After initial null effect, a slight positive impact was seen in all 
the gold funds while marked positive response occurred in funds $\mathrm{f} \_1, \mathrm{f} \_15, \mathrm{f} \_16$, f_17, f_18, f_19, f_20, f_22, f_23, f_25 \& f_26. A Negligible effect was recorded when impulse was given to energy funds.

Interest rate impulses showed a positive impact on all gold funds leading to a raised NAV by the end of the $10^{\text {th }}$ period. After initial null response, fund $f_{-} 1$, $\mathrm{f} \_15, \mathrm{f} \_16, \mathrm{f} \_17, \mathrm{f} \_19, \mathrm{f} \_20, \mathrm{f} \_22, \mathrm{f} \_23, \mathrm{f} \_25 \mathrm{\&} \mathrm{f} \_26$ showed very huge positive effect raising the NAVs. Energy funds, however, showed negligible fluctuation in NAVs.

An impulse in Sensex caused a negligible effect on all the gold funds while all the energy funds showed a negative effect. In $\mathrm{f} \_27 \& \mathrm{f} \_32$ the effect was more pronounced.

An impulse in Nifty caused an immediate null effect on all the funds, but gradually a negative response set in for most of the funds finishing towards lower NAVs towards the end of the $10^{\text {th }}$ period. Some gold funds like $f \_16, f \_17$, f_19, f_20, f_22, f_23, f_25 \& f_26 showed no immediate effect, gradually showed declining NAVs and towards the end recovered a little settling down to less negative NAVs.

\subsection{Variance Decomposition (Table 3)}

On doing variance decomposition (Table 3) of funds NAVs, we see that none of the macroeconomic variables has an instantaneous effect. It is only own innovations that generate $100 \%$ variance in the $1^{\text {st }}$ period. Effect of fund's own innovations starts decreasing over time and generates about 50\% - 60\% effect in all the funds till the end of the $10^{\text {th }}$ period.

At the end of the $10^{\text {th }}$ period, the gold funds showed an impact of around 20\% - $25 \%$ when an innovation in interest rates was introduced. About $50 \%$ of the sample gold funds showed a marked impact of the impulse of foreign exchange reserves. Energy funds on an average showed a good response to a shock in money supply leading to $15 \%-20 \%$ impact on NAVs. Though $\mathrm{f} \_28$ showed no change.

Rest all the macroeconomic impulses showed marginal diminishing effects on various funds.

\section{Discussion of Results}

The whole world is interconnected and no country's economy is isolated from that of another. If any change takes place anywhere its ripples are felt all over the globe be it terrorism, stock market crash or any political event. We saw how the changes in macroeconomic variables caused sudden changes in stock markets. Own fund information led to a high fluctuation in mutual fund NAV's, probably due to change in investor perception about the future profits and losses from the said fund. A good fund information would increase the investment in mutual funds, thus raising their NAV's while vice versa will happen when an unfavorable fund information pours in. 
Table 3. Variance decomposition.

\begin{tabular}{|c|c|c|c|c|c|c|c|c|c|c|}
\hline & $\begin{array}{l}\text { Fund's own } \\
\text { innovation }\end{array}$ & $\begin{array}{c}\text { Innovation } \\
\text { in } M \_1\end{array}$ & $\begin{array}{c}\text { Innovation } \\
\text { in } \mathbf{M} \_2\end{array}$ & $\begin{array}{c}\text { Innovation } \\
\text { in } M \_3\end{array}$ & $\begin{array}{c}\text { Innovation } \\
\text { in } M \_4\end{array}$ & $\begin{array}{c}\text { Innovation } \\
\text { in } M \_5\end{array}$ & $\begin{array}{c}\text { Innovation } \\
\text { in } M \_6\end{array}$ & $\begin{array}{c}\text { Innovation } \\
\text { in } M \_7\end{array}$ & $\begin{array}{c}\text { Innovation } \\
\text { in } M_{-} 8\end{array}$ & $\begin{array}{c}\text { Innovation } \\
\text { in } M \_9\end{array}$ \\
\hline f_1 & 47 & 2.5 & 1.3 & 2.7 & 2 & 1 & 8.7 & 32 & 0.5 & 1.6 \\
\hline f_2 & 60 & 3 & 0.3 & 0.7 & 1.4 & 0.7 & 2 & 25 & 1.6 & 5 \\
\hline f_3 & 65 & 2 & 0.5 & 1.7 & 0.7 & 0.5 & 6 & 20 & 1 & 4 \\
\hline f_4 & 56 & 0.7 & 1.3 & 3 & 0.5 & 0.8 & 11 & 26 & 0.2 & 0.6 \\
\hline f_5 & 61 & 1.3 & 0.1 & 0.9 & 1.7 & 0.5 & 4 & 24 & 1.4 & 5 \\
\hline f_6 & 60 & 1.6 & 0.5 & 0.6 & 1.8 & 0.8 & 2.9 & 23 & 1.9 & 8 \\
\hline f_7 & 44 & 1.7 & 0.6 & 0.4 & 2 & 2 & 23 & 21 & 1.5 & 3 \\
\hline f_8 & 68 & 3.6 & 0.2 & 0.6 & 0.5 & 0.4 & 4 & 16 & 2 & 4 \\
\hline f_9 & 57 & 1 & 1 & 1 & 3.5 & 0.7 & 2.7 & 26 & 1.3 & 4.7 \\
\hline f_10 & 56 & 0.9 & 1 & 1.3 & 4 & 1 & 4 & 24.7 & 1 & 5.7 \\
\hline f_11 & 57 & 1.7 & 0.6 & 1.5 & 3 & 0.7 & 3 & 25.5 & 1 & 5 \\
\hline $\mathrm{f} \_12$ & 65 & 1.8 & 0.4 & 2 & 6.5 & 0.8 & 13.5 & 3.7 & 1 & 5 \\
\hline f_13 & 70.6 & 2 & 1.8 & 8 & 6 & 0.5 & 7 & 1 & 0.1 & 2 \\
\hline f_14 & 62.5 & 0.3 & 0.3 & 1 & 5 & 2 & 2.3 & 20 & 1.4 & 5 \\
\hline f_15 & 42 & 1.8 & 0.6 & 0.4 & 2 & 2 & 25 & 20.8 & 1.6 & 3 \\
\hline$f \_16$ & 43 & 1.7 & 0.6 & 0.4 & 2 & 2 & 25 & 20.6 & 1.5 & 3 \\
\hline f_17 & 50 & 1.3 & 1 & 1 & 5.3 & 1 & 9 & 28 & 1 & 2.5 \\
\hline f_18 & 54 & 3 & 1 & 0.7 & 0.8 & 2.3 & 10 & 27 & 0.3 & 0.8 \\
\hline f_19 & 43 & 1.8 & 0.6 & 0.4 & 2 & 2 & 25 & 20 & 1.5 & 3 \\
\hline f_20 & 43 & 1.8 & 0.5 & 0.4 & 2 & 2 & 24.6 & 20 & 1.6 & 3 \\
\hline f_21 & 42 & 1.7 & 0.6 & 0.4 & 2 & 2 & 25 & 21 & 1.6 & 3 \\
\hline f_22 & 43 & 1.8 & 0.6 & 0.4 & 2 & 2 & 24 & 20 & 1.5 & 3 \\
\hline f_23 & 44 & 1.6 & 0.5 & 1.6 & 1.3 & 2 & 15 & 30 & 0.9 & 2.8 \\
\hline f_24 & 43 & 1.8 & 0.6 & 0.4 & 2 & 2.3 & 25 & 21 & 1.5 & 3 \\
\hline f_25 & 43 & 1.7 & 0.6 & 0.4 & 2 & 2.2 & 24 & 21 & 1.6 & 3 \\
\hline f_26 & 43 & 1.8 & 0.6 & 0.4 & 2 & 2 & 24.3 & 21 & 1.5 & 3 \\
\hline f_27 & 42 & 5 & 11 & 7.8 & 0.3 & 11.8 & 1.6 & 1.7 & 15 & 3.7 \\
\hline f_28 & 83.6 & 5 & 0.1 & 10 & 1 & 0.3 & 2 & 1.5 & 5 & 0.5 \\
\hline f_29 & 43 & 9 & 18 & 2.5 & 2 & 12 & 2.7 & 1.4 & 6 & 3 \\
\hline f_30 & 50 & 3.5 & 3 & 11.6 & 0.8 & 16.6 & 0.1 & 1.7 & 5 & 8 \\
\hline f_31 & 38 & 3 & 6.3 & 12.4 & 2.4 & 23.8 & 0.7 & 2.5 & 3 & 7.5 \\
\hline f_32 & 46 & 2.7 & 1.4 & 11.9 & 0.6 & 12.4 & 0.8 & 1.6 & 17.9 & 4.8 \\
\hline f_33 & 53.6 & 11 & 5.8 & 1.8 & 2.4 & 12 & 0.9 & 1.3 & 2.5 & 8.5 \\
\hline f_34 & 45 & 7 & 3.5 & 6 & 0.2 & 12.7 & 0.8 & 1.6 & 13 & 10 \\
\hline f_35 & 32.5 & 13.4 & 1.6 & 6.3 & 0.3 & 15 & 0.4 & 1 & 9 & 20 \\
\hline f_36 & 26.8 & 7.8 & 6.7 & 10 & 0.6 & 21 & 2.2 & 0.7 & 2.7 & 22 \\
\hline f_37 & 51 & 7 & 2 & 16 & 0.2 & 12.6 & 0.8 & 0.2 & 2.3 & 7.8 \\
\hline f_38 & 42.5 & 5 & 2.3 & 17.8 & 0.4 & 19 & 0.2 & 2.2 & 6 & 4.7 \\
\hline f_39 & 50 & 3.8 & 9.3 & 9 & 1.5 & 10.7 & 0.7 & 1.8 & 1.7 & 11.5 \\
\hline f_40 & 44 & 7.5 & 5 & 7.6 & 0.1 & 15.8 & 0.6 & 0.6 & 4 & 14.4 \\
\hline f_41 & 56.7 & 7.8 & 2.8 & 2.6 & 1.5 & 15.8 & 0.2 & 0.7 & 5.4 & 6.5 \\
\hline
\end{tabular}

Source: researcher's analysis [17] [18]. 
In case of interest rate changes, investors usually look for alternative investment avenues, where they expect profits. Hence, it was seen that when there was a change in interest rate, more investments and hence higher NAV's were reported in case of gold funds. Less effect was seen in case of energy funds, as probably investors perceive "gold" funds less volatile than "energy" funds. Energy sector as such has a trend of high fluctuation. It was also seen that as foreign exchange reserves changed, it led to changed (increased) investments in gold funds. The probable reason could be that whenever foreign exchange reserves change, there is a change in the strength of rupee. This instantly affects the investor psychology, who start looking for safe alternative investment avenues. Low degree of cointegration with all macroeconomic variables was reported when energy mutual funds were considered. This may be because these funds are a relatively new concept and investors have not developed high confidence in them. However, it was seen that whenever there was an increase in money supply, investors increased investments in energy funds, since probably they considered "energy funds" good investment avenues to park their extra savings.

USD exchange rate, crude oil price, silver price, gold price, Sensex and Nifty were seen to have a diminishing impact on most of the funds, the reason could be attributed to the already set in turbulent world economic situation since past many years now.

In such a situation the common investor is left at the mercy of stock markets. The rupee has been depreciating for some time now and the trend is almost constant. Crude oil remains one of the most important variables in the world economy even after its prices have fallen to all-time low by February 2016. The most important reason for this is that the "supply" of crude is limited and after the unrest in West Asian and Arabic world, the situation has worsened. The USA has been exporting oil but we know there is a limit to it, the crude formation in the USA is very deep inside and its drilling is a costly affair. Post-Chinese market crash last year, the demand for crude oil declined, which rendered it cheaper. Post Brexit it is predicted that crude and other commodity prices will slacken down. For India this is seen as a positive aspect since last time the crude oil prices crashed, inflation came under control. Also, since the investments abroad will get lesser, the common investor is believed to be switching over to assets like gold so as to play safe. Since physical gold is also prone to all these macroeconomic factors, the best option in this situation is to invest in portfolio of assets like in mutual funds.

Though the research presented here was done carefully taking all precautions, yet there are certain limitations and scope for further research. Only nine macroeconomic variables were chosen based on the frequency of data availability. Other variables that effect macroeconomy may be included, which have low data frequency, by expanding the time period of study. These factors can include GDP (gross domestic product), CPI (consumer price index), WPI (wholesale price index), IIP (index of industrial production), FDI (foreign direct invest- 
ment), FII (foreign institutional investment), Per capita income etc. Funds other than energy and gold funds can also be investigated, which may include money market funds.

\section{Conclusions}

We accept $\mathrm{Ho}_{3}, \mathrm{Ho}_{5}$, and $\mathrm{Ho}_{8}$ when we consider the case of gold funds as the gold price, money supply, and Sensex have an insignificant effect on gold funds in long run. But in short run money supply had a little negative impact. We reject $\mathrm{Ho}_{1}$ and $\mathrm{Ho}_{5}$ and accept all other hypothesis in case of energy funds since only money supply and USD exchange rate were found to have a profound impact both in long term and short term.

On the whole, it can be said that the various macroeconomic variables impact gold and energy mutual funds. The variables impact each other significantly thus leading to changes in the global supply, demand, and prices. The impulses start from the global and national stage and slowly penetrate deeply into the investment market. The common investor must be aware of this interplay so as to make a wise decision of investment. We have seen how some variables particularly impact gold funds and some variables particularly impact the energy funds. It may also be that when one category of fund is affected badly, the other may get an advantage and show a boost in their NAVs. Thus it is important to keep a track of all the macroeconomic happenings in the country and the world at large so as to avoid missing opportunities.

\section{Conflicts of Interest}

The authors declare no conflicts of interest regarding the publication of this paper.

\section{References}

[1] Ahmad, K.M., Ashraf, S. and Ahmed, S. (2005) Is the Indian Stock Market Integrated with the US and Japanese Markets? An Empirical Analysis. South Asia Economic Journal, 6, 193-206. https://doi.org/10.1177/139156140500600202

[2] Gera, A. (2007) Macro-Economic Factors Affecting Mutual Funds in India \& Basis for Evaluating Mutual Fund Performance. Alliance Business School.

[3] Dash, M. and Kumar, G.D. (2008) A Study on the Effect of Macroeconomic Variables on Indian Mutual Funds. http://ssrn.com/abstract $=1316442$

[4] Gupta, P.K. and Siddiqui, S. (2010) Weak Form of Market Efficiency: Evidences from Selected NSE Indices.

[5] Srivastava, A. (2010) Relevance of Macroeconomic Factors for the Indian Stock Market. Decision, 37, 69-89.

[6] Shukla, S. (2011) Role of Macroeconomic Variables in Indian Mutual Fund Industry. Analytique, 7, 4-13.

[7] Khalid, M. (2012) Long-Run Relationship between Macroeconomic Variables and Stock Return: Evidence from Karachi Stock Exchange (KSE). School of Doctoral Studies (European Union) Journal, 95-105.

[8] Patel, S. (2012) The Effect of Macroeconomic Determinants on the Performance of 
the Indian Stock Market. NMIMS Management Review, 22, 117-127.

[9] Joshi, M. (2013) Factors Affecting Indian Stock Market. International Journal of Contemporary Research in Management, Engineering and Health Science, 2, 37-45. https://doi.org/10.2139/ssrn.2238539

[10] Singhania, M. and Anchalia, J. (2013) Volatility in Asian Stock Markets and Global Financial Crisis. Journal of Advances in Management Research, 10, 333-351. https://doi.org/10.1108/JAMR-01-2013-0010

[11] Abdul, N.B. and Sarvani, K. (2014). Evaluating the Performance of Indian Mutual Funds Schemes: Macroeconomic Factors as Performance Indicators. In: 11 th Asian Business Research Conference, BIAM Foundation, Dhaka. http://www.wbiworldconpro.com/pages/paper/dhaka-conference-2014/2318

[12] Kumar, R. and Gautam, S. (2014) An Empirical Study on Impact of FII and Other Stock Exchanges Volatility on BSE Stock Exchange Volatility'. International Journal of Engineering Technology, Management and Applied Sciences, 2.

[13] Sanningammanavara, K.V., Kumar, K. and Rakesh, H.M. (2014) Macro-Economic Forces and Indian Stock Market: An Empirical Relation. International Journal of Commerce, Business and Management, 3, 457-465.

[14] Singh, P. (2014) Indian Stock Market and Macroeconomic Factors in Current Scenario. International Journal of Research in Business Management, 2, 43-54.

[15] Choudhary, N., Nair, G.K. and Purohit, H. (2015) Volatility in Copper Prices in India. Annals of Financial Economics, 10, 1-26. https://doi.org/10.1142/S2010495215500086

[16] Mohanasundari, M. and Vidhyapriya, P. (2015) Indian Mutual Fund Industry: An Impact of Investor's Perception and Performance of Tax Saving Schemes in Mutual Fund. International Business Management, 9, 267-272.

[17] Hair, J.F. and Anderson, R.E. (2006) Multivariate Data Analysis. 6th Edition, Pearson Education, New Delhi.

[18] Nargundkar, R. (2008) Marketing Research: Text and Cases. 3rd Edition, Tata McGraw Hill, New Delhi. 


\section{Appendix}

Table A1. Coding.

\begin{tabular}{|c|c|}
\hline \multicolumn{2}{|l|}{ Mutual Funds Code } \\
\hline$R^{\star}$ Shares Gold Etf Dividend (Nav) & F_1 \\
\hline Axis Gold Fund Rg (Nav) & F_2 \\
\hline Birla Sun Life Gold Fund Rg (Nav) & F_3 \\
\hline Canara Robeco Gold Savings Fund Rg (Nav) & F_4 \\
\hline Hdfc Gold Fund (Nav) & F_5 \\
\hline Icici Prudential Regular Gold Savings Fund Rg (Nav) & F_6 \\
\hline Idbi Gold Fund Rg (Nav) & F_7 \\
\hline Invesco India Gold Fund Rg (Nav) & F_8 \\
\hline Kotak Gold Fund G (Nav) & F_9 \\
\hline Reliance Gold Savings Fund G (Nav) & F_10 \\
\hline Sbi Gold Fund Rg (Nav) & F_11 \\
\hline Dsp Br World Gold Fund Regular G (Nav) & F_12 \\
\hline Kotak World Gold Fund Std. G (Nav) & F_13 \\
\hline Quantum Gold Saving Fund G (Nav) & F_14 \\
\hline Quantum Gold Fund Etf (Nav) & F_15 \\
\hline Axis Gold Etf (Nav) & F_16 \\
\hline Birla Sun Life Gold Etf (Nav) & F_17 \\
\hline Canara Gold Etf (Nav) & F_18 \\
\hline Gs Gold Bees (Nav) & F_19 \\
\hline Hdfc Gold Etf (Nav) & F_20 \\
\hline Icici Pru Gold Iwin Etf (Nav) & F_21 \\
\hline Idbi Gold Etf (Nav) & F_22 \\
\hline Invesco India Gold Etf (Nav) & F_23 \\
\hline Kotak Gold Etf (Nav) & F_24 \\
\hline Sbi Etf Gold (Nav) & F_25 \\
\hline Uti Gold Etf (Nav) & F_26 \\
\hline Uti Growth Sectors-Energy Fund G (Nav) & F_27 \\
\hline Dsp Br World Energy Fund Rg (Nav) & F_28 \\
\hline Dsp Black Rock Natural Resources And New Energy Fund Rp-G (Nav) & F_29 \\
\hline Reliance Diversified Power Sector Fund-G (Nav) & F_30 \\
\hline Escorts Power And Energy Fund Rg (Nav) & F_31 \\
\hline Sahara Power And Natural Resources G (Nav) & F_32 \\
\hline Sbi Infrastructure Fund Rg (Nav) & F_33 \\
\hline Sahara Infrastructure Fund Var Rg (Nav) & F_34 \\
\hline Canara Robeco Infrastructure Fund G (Nav) & F_35 \\
\hline
\end{tabular}




\begin{tabular}{cc} 
Continued & \\
\hline Taurus Infrastructure Fund Rg (Nav) & F_36 \\
Baroda Pioneer Infrastructure Fund G (Nav) & F_37 \\
Escorts Infrastructure Fund G (Nav) & F_38 \\
Idfc Infrastructure Fund Gr (Nav) & F_39 \\
Icici Prudential Infrastructure Fund G (Nav) & F_40 \\
Invesco India Infrastructure Fund Rg (Nav) & F_41 \\
MACROECONOMIC VARIABLES & \\
Indian rupee spot exchange rate M_1 & \\
Brent Crude Futures & Gold Spot \\
Silver Spot Price & M_2 \\
India Money Supply M3 Yoy & M_3 \\
Indian Foreign Exchange Reserves Usd & M_4 \\
91 Day Treasury Bill Yield & M_5 \\
Sensex & M_6 \\
Nifty 50 & M_7 \\
\end{tabular}

Table A2. Coding groups.

\begin{tabular}{|c|c|}
\hline Group & Members \\
\hline Group 1 & f_1, M_1, M $\_2, M \_3, M \_4, M \_5, M \_6, M \_7, M \_8, M \_9$ \\
\hline Group 2 & f_2, M_1, M_2, M_3, M $\_4, M \_5, M \_6, M \_7, M \_8, M \_9$ \\
\hline Group 3 & f_3, M_1, M $\_2, M \_3, M \_4, M \_5, M \_6, M \_7, M \_8, M \_9$ \\
\hline Group 4 & f_4, M_1, M_2, M_3, M_4, M_5, M_6, M_7, M_8, M_9 \\
\hline Group 5 & f_5, M_1, M_2, M_3, M_4, M_5, M_6, M_7, M_8, M_9 \\
\hline Group 6 & f_6, M_1, M $\_2, M \_3, M \_4, M \_5, M \_6, M \_7, M \_8, M \_9$ \\
\hline Group 7 & 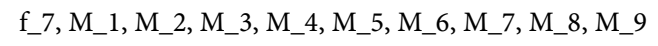 \\
\hline Group 8 & f_8, M_1, M_2, M_3, M $\_4, M \_5, M \_6, M \_7, M \_8, M \_9$ \\
\hline Group 9 & 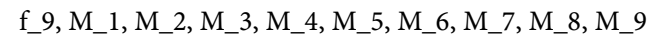 \\
\hline Group 10 & 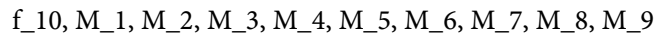 \\
\hline Group 11 & $f \_11, M \_1, M \_2, M \_3, M \_4, M \_5, M \_6, M \_7, M \_8, M \_9$ \\
\hline Group 12 & $f \_12, M \_1, M \_2, M \_3, M \_4, M \_5, M \_6, M \_7, M \_8, M \_9$ \\
\hline Group 13 & $f \_13, M \_1, M \_2, M \_3, M \_4, M \_5, M \_6, M \_7, M \_8, M \_9$ \\
\hline Group 14 & $f \_14, M \_1, M \_2, M \_3, M \_4, M \_5, M \_6, M \_7, M \_8, M \_9$ \\
\hline Group 15 & $f \_15, M \_1, M \_2, M \_3, M \_4, M \_5, M \_6, M \_7, M \_8, M \_9$ \\
\hline Group 16 & $f \_16, M \_1, M \_2, M \_3, M \_4, M \_5, M \_6, M \_7, M \_8, M \_9$ \\
\hline Group 17 & $f \_17, M \_1, M \_2, M \_3, M \_4, M \_5, M \_6, M \_7, M \_8, M \_9$ \\
\hline Group 18 & $f \_18, M \_1, M \_2, M \_3, M \_4, M \_5, M \_6, M \_7, M \_8, M \_9$ \\
\hline Group 19 & f_19, M_1, M $\_2, M \_3, M \_4, M \_5, M \_6, M \_7, M \_8, M \_9$ \\
\hline
\end{tabular}




\section{Continued}

Group 20

Group 21

Group 22

Group 23

Group 24

Group 25

Group 26

Group 27

Group 28

Group 29

Group 30

Group 31

Group 32

Group 33

Group 34

Group 35

Group 36

Group 37

Group 38

Group 39

Group 40

Group 41 f_20, M_1, M_2, M_3, M_4, M_5, M_6, M_7, M_8, M_9

f_21, M_1, M_2, M_3, M_4, M_5, M_6, M_7, M_8, M_9

f_22, M_1, M_2, M_3, M_4, M_5, M_6, M_7, M_8, M_9

f_23, M_1, M_2, M_3, M_4, M_5, M_6, M_7, M_8, M_9

f_24, M_1, M $\_2$, M_3, M_4, M_5, M_6, M_7, M_8, M_9

f_25, M_1, M_2, M_3, M_4, M_5, M_6, M_7, M_8, M_9

f_26, M_1, M_2, M_3, M_4, M_5, M_6, M_7, M_8, M_9

f_27, M_1, M_2, M_3, M_4, M_5, M_6, M_7, M_8, M_9

f_28, M_1, M_2, M_3, M_4, M_5, M_6, M_7, M_8, M_9

f_29, M_1, M_2, M_3, M_4, M_5, M_6, M_7, M_8, M_9

f_30, M_1, M_2, M_3, M_4, M_5, M_6, M_7, M_8, M_9

f_31, M_1, M_2, M_3, M_4, M_5, M_6, M_7, M_8, M_9

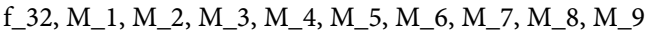

f_33, M_1, M_2, M_3, M_4, M_5, M_6, M_7, M_8, M_9

f_34, M_1, M_2, M_3, M_4, M_5, M_6, M_7, M_8, M_9

f_35, M_1, M_2, M_3, M_4, M_5, M_6, M_7, M_8, M_9

f_36, M_1, M_2, M_3, M_4, M_5, M_6, M_7, M_8, M_9

f_37, M_1, M_2, M_3, M_4, M_5, M_6, M_7, M_8, M_9

f_38, M_1, M_2, M_3, M_4, M_5, M_6, M_7, M_8, M_9

f_39, M_1, M_2, M_3, M_4, M_5, M_6, M_7, M_8, M_9

f_40, M_1, M_2, M_3, M_4, M_5, M_6, M_7, M_8, M_9

f_41, M_1, M_2, M_3, M_4, M_5, M_6, M_7, M_8, M_9 